\title{
Kinematic characteristics of the scapula and clavicle during military press exercise and shoulder flexion.
}

\section{AUTHOR(S):}

Ichihashi, Noriaki; Ibuki, Satoko; Otsuka, Naoki; Takashima, Shingo; Matsumura, Aoi

\section{CITATION:}

Ichihashi, Noriaki ...[et al]. Kinematic characteristics of the scapula and clavicle during military press exercise and shoulder flexion.. Journal of shoulder and elbow surgery 2014, 23(5): 649-657

\section{ISSUE DATE:}

2014-05

URL:

http://hdl.handle.net/2433/187360

\section{RIGHT:}

(C) 2014 Journal of Shoulder and Elbow Surgery Board of Trustees. Published by Mosby, Inc; This is not the published version. Please cite only the published version.; この論文は 出版社版でありません。引用の際には出版社版をご確認ご利用ください。 
Kinematic characteristics of the scapula and clavicle during military press exercise and shoulder flexion

Running title: Scapula and clavicle kinematic characteristics of military press

Noriaki Ichihashi, PT, $\mathrm{PhD}^{\mathrm{a}}$

Satoko Ibuki, PT

Naoki Otsuka, $\mathrm{PT}^{\mathrm{b}}$

Shingo Takashima, PT, MS ${ }^{\mathrm{b}}$

Aoi Matsumura, PT,MS

${ }^{a}$ Human Health Sciences, Graduate School of Medicine, Kyoto University, Kyoto, Japan

${ }^{\mathrm{b}}$ ASICS Corporation, Institution of Sport Science, Kobe, Japan

${ }^{\mathrm{c}}$ Kyoto University Hospital

* Corresponding author:

Noriaki Ichihashi

Human Health Sciences, Graduate School of Medicine, Kyoto University

53 Kawara-cho, Shogoin, Sakyo-ku, Kyoto 606-8507, Japan

Tel: $+81-75-751-3951$

Fax: +81-75-751-3951

E-mail: tokumitsu.satoko.6c@kyoto-u.ac.jp

Conflict of Interest Statement

None of the authors has any conflict of interest associated with this study. 
This study has been approved by the Ethics Committee of the Kyoto University Graduate School and Faculty of Medicine (E1192) 
Scapula and clavicle kinematic characteristics of military press

Kinematic characteristics of the scapula and clavicle during military press exercise and shoulder flexion

\section{$1 \quad$ Abstract}

\section{$2 \quad$ Background}

3 The military press is an exercise frequently prescribed for scapular and shoulder

4 rehabilitation. Although this exercise has previously been analyzed

5 electromyographically, its kinematic features remain poorly understood. In the present

6 study, we aimed to clarify these features of the military press and suggest relevant clinical

7 applications.

8 Methods

9 Sixteen healthy males participated in this study. The participants performed the military

10 press while holding $2 \mathrm{~kg}$ weights as well as shoulder flexion with and without 2-kg weights,

11 and an electromagnetic motion capture system was used to analyze the kinematic features

12 of the scapula, clavicle, and humerus during these exercises. The motions of the scapula

13 and clavicle were analyzed at $10^{\circ}$ increments of shoulder flexion from $30^{\circ}$ to $120^{\circ}$.

Results

16 The military press involved less scapular internal rotation, greater upward rotation, and

17 greater posterior tilt than shoulder flexion with or without weights, especially in the starting

18 to middle range of shoulder flexion. Greater clavicular retraction and elevation were also

19 seen during the military press.

\section{Discussion}


Scapula and clavicle kinematic characteristics of military press

21 The movements of the scapula and clavicle during the military press differ significantly

22 from those during shoulder flexion with and without weights. The kinematic features of

23 the military press, which involved less scapular internal rotation, greater upward rotation,

24 and greater posterior tilt than did shoulder flexion, may make it a useful re-education

25 exercise (if pain allows) for patients with decreased scapular external rotation, upward

26 rotation, and posterior tilting. The results of this study might provide a kinematic basis

27 for the use of this widely performed shoulder exercise.

28

29 Keywords: scapular motion, biomechanics, rehabilitation, military press, shoulder flexion,

30 multi-joint movement. 
Scapula and clavicle kinematic characteristics of military press

\section{Introduction}

The military press is an exercise frequently used in scapular and shoulder rehabilitation. . $^{2,7,10,21,26,29}$ It is a variation of an overhead press, which elevates the humerus overhead from an initial position with the elbow flexed and positioned anterior to the shoulder. Most of the previous studies on this exercise have involved electromyographic (EMG) analysis. Townsend ${ }^{29}$ analyzed the activities of the rotator cuff, deltoid, pectoralis major, and latissimus dorsi muscles of the shoulder during shoulder exercises, including the military press. They suggested that the supraspinatus, subscapularis, and anterior and middle deltoid muscles are highly active during the military press. Moseley et al. ${ }^{21}$ studied the activities of the scapular muscles during the military press and other rehabilitation exercises and suggested that the military press is a useful exercise for the upper trapezius, middle serratus anterior, and lower serratus anterior muscles.

On the other hand, the kinematic features of this exercise are not well understood.

Crenshaw et al. indicated that the overhead pressing motion involved in the military press can decrease the amount of space in the subacromial area and thereby increase the stress on the subacromial space in throwing athletes who have preexisting chronic changes in this space. ${ }^{4}$ However, it is also true that in clinical situations, there are many patients with shoulder complications (such as impingement, labral injury, and frozen shoulder) who can elevate their arms (or weights) more easily during military press than during shoulder flexion. Although many kinematic analyses have been performed on humeral elevation in various planes such as abduction, ${ }^{16}$ scaption, ${ }^{1,19,28}$ and flexion, ${ }^{16}$ all of these analyses were performed with the arms fully extended. To our knowledge, there is no kinematic study of the military press, i.e., humeral elevation accompanied by active elbow movement. In the present study, using an electromagnetic sensor, we aimed to investigate the 
Scapula and clavicle kinematic characteristics of military press

58 three-dimensional kinetic features of the military press in comparison with those of

59 shoulder flexion, performed with and without weights, in order to clarify the clinically

60 relevant characteristics of this exercise. We hypothesize that the military press has

61 kinematic features such as greater scapular upward rotation, posterior tilt, and external

62 rotation that could make it a better humeral elevation exercise than normal shoulder flexion

63 with the elbow extended.

64 
Scapula and clavicle kinematic characteristics of military press

\section{Materials and Methods}

\subsection{Participants}

Sixteen healthy males (age, $21.8 \pm 1.1$ (mean $\pm \mathrm{SD}$ ) years; height, $173.3 \pm 5.3 \mathrm{~cm}$; and weight, $62.9 \pm 7.3 \mathrm{~kg}$ ) participated in this study. Subjects with a previous history of upper limb surgery, a present or previous history of neuromuscular disease, or any complaint in the upper limb in the past year were excluded from the study. The participants' dominant limbs were analyzed.

\subsection{Instrumentation}

Three-dimensional kinematic data for the scapula, clavicle, and humerus were recorded using a 6-degrees-of-freedom electromagnetic motion capture system (Liberty; Polhemus). The Polhemus Liberty system consists of a transmitter and sensors. Its System Electronics Unit generates and senses the magnetic fields and computes the position and orientation of each sensor. Previous studies have demonstrated the accuracy of this device for the measurement of upper limb motion. For angles of shoulder flexion less than $120^{\circ}$, the error of measurement of the scapula and clavicle (relative to measurements made using bone pins) is less than $5^{\circ} \cdot{ }^{12,15,16,18}$ Therefore, only the data corresponding to shoulder flexion angles up to $120^{\circ}$ were analyzed in this study.

The transmitter was fixed on a rigid wooden board and the global coordinate system (GCS) was established. The sensors were fixed to the skin overlying the flat surface of the superior acromion process, the sternum, and the humerus (via a molded thermoplastic cuff at the midpoint of the humerus). Next, the bony landmarks of the scapula, clavicle, and humerus were palpated and then digitized using the LIBERTY sensor STYLUS to establish the anatomically based local coordinate systems (LCS). These measurements were performed with the subjects standing still with their arms hanging 
Scapula and clavicle kinematic characteristics of military press

90 beside their bodies. Each LCS was defined according to the International Society of

91 Biomechanics (ISB) standardization proposal for the upper extremity ${ }^{3233}$ (Figure 1). The

92

93

94 acromial angle (AA), trigonum spinae (TS), and inferior angle (IA) were used to define the LCS of the scapula. The scapular SX axis was directed from the TS to the AA. The SY axis was perpendicular to the plane defined by the TS, AA, and IA, and the SZ axis was defined as the cross product of the SX and SY.

The xiphoid process (XP), suprasternal notch (SN), spinous process of the seventh cervical vertebra (C7), and spinous process of the eighth thoracic vertebra (T8) were used to define the LCS of the thorax. The thoracic TZ (vertical axis) was directed from the midpoint of the T8 and XP to the midpoint of the $\mathrm{SN}$ and $\mathrm{C7}$; the $\mathrm{TX}$ (transverse axis) was perpendicular to the plane defined by the $\mathrm{SN}, \mathrm{C} 7, \mathrm{~T} 8$, and the $\mathrm{XP}$; and the TY (sagittal axis) was defined as the cross product of the TZ and TX. The medial epicondyle (ME), lateral epicondyle (LE), and glenohumeral joint center $(\mathrm{GH})$ were digitized to define the humeral coordinate system. The humeral HZ (longitudinal axis) was directed from the midpoint of the ME and LE to the GH; the HY (anterior-directed axis) was perpendicular to the plane defined by the GH, ME, and LE; and the HX (laterally directed axis) was defined as the cross product of the HY and HZ. The acromioclavicular joint (AC) and sternoclavicular joint (SC) were used to define the LCS of the clavicle. The clavicular CX axis was directed from the AC to the SC. The thoracic $\mathrm{TZ}$ axis was also used as the $\mathrm{CZ}$ axis, and the $\mathrm{CY}$ axis was defined as the cross product of the $\mathrm{CX}$ and $\mathrm{CZ}$.

\subsection{Procedures}

The kinematic features of the shoulder were analyzed during the following three exercises: shoulder flexion without weights, shoulder flexion while holding 2-kg weights in both hands, and the military press while holding 2-kg weights in both hands (Figure 2). 
Scapula and clavicle kinematic characteristics of military press

115 All three exercises were performed with the subject sitting on a platform without any

116 backrest or armrest. In all three motions, humeral elevation was performed in the sagittal

117 plane. In the starting position, the arms were allowed to hang at the sides with the elbow

118 extended for shoulder flexion, and with the elbow fully flexed for the military press. The

119 subjects raised their arms to full elevation over the course of four seconds, using a

120 metronome for speed control. Each exercise was performed five times consecutively, and

121 the mean of the middle three elevations was analyzed. The patients were sufficiently

122 rested before performing the next exercise, and the order in which the exercises were

123 performed was randomized for each participant to avoid any effect of the testing order.

124 For the military press, the subjects were instructed to keep the elbow in the plane of flexion.

125 In our previous study, ${ }^{22}$ the repeat-trial ICC values for the kinematic data for the scapula and clavicle ranged from 0.94 to 0.99 , indicating almost-perfect reliability

\subsection{Data reduction}

The raw kinematic data were filtered using a low-pass 4-Hz Butterworth filter.

The rotations of the distal coordinate system were described with respect to the proximal coordinate system using Euler angles in accordance with the recommendations of the International Shoulder Group of the International Society of Biomechanics (ISB) ${ }^{32}$ (Figure 3). The rotational motion of the scapula relative to the thorax was defined as follows.

134 The motion of the scapula around the SZ axis was defined as external rotation

135 (negative)/internal (positive) rotation, the motion around the SY axis was defined as upward rotation (negative)/downward rotation (positive), and the motion around the SX axis was defined as anterior tilt(negative)/posterior tilt (positive). The rotation of the clavicle relative to the thorax was defined as follows. The motion of the clavicle around the $\mathrm{CZ}$ axis was defined as protraction (negative) or retraction (positive). The motion of 
Scapula and clavicle kinematic characteristics of military press

140 the clavicle around the CY axis was defined as elevation (negative) or depression (positive).

141 The motion of the humerus relative to the thorax was defined as the elevation angle,

142 wherein a positive value represented elevation. The scapular angle (upward/downward

143 rotation, external/internal rotation, and posterior/anterior tilting) and clavicular angle

144 (protraction/retraction and elevation/depression) were measured at selected humeral

145 elevation angles during the ascending phase of each task using custom Matlab (Mathworks,

146 Inc., USA) code. For the descriptive portion of the study, humeral angles relative to the

147 thorax were selected at $10^{\circ}$ intervals and ranged from $30^{\circ}$ to $120^{\circ}$ of humeral elevation.

2.5 Data analysis

The means of the middle three trials were analyzed. Two-way (exercise type

151 [flexion without weight/flexion with weight/military press] $\times$ humeral elevation angle)

152 repeated-measures analysis of variance (ANOVA) was used to analyze differences in the

153 scapular and clavicular angles. Differences were considered statistically significant at $\mathrm{p}<$

154 0.05. When a significant main effect or any interaction of the exercise type was found,

155 post-hoc analysis with Holm adjustment was used to assess the significance of differences between the individual types of exercise. 
Scapula and clavicle kinematic characteristics of military press

158

159

160

161

162

163

164

165

166

167

168

169

170

171

172

\section{Results}

\subsection{Scapular Internal/External Rotation (Table 1, Figure 4A,)}

The exercise type had a significant main effect on the scapular internal/external rotation angle $(\mathrm{p}<0.01)$, with a significant interaction noted between the exercise type and the humeral elevation angle $(\mathrm{p}<0.01)$. Post-hoc analysis with Holm adjustment revealed significantly less scapular internal rotation during the military press than during shoulder flexion with or without weights at humeral elevation angles of $30^{\circ}$ to $100^{\circ}(\mathrm{p}<0.05)$.

3.2. Scapular Upward/Downward Rotation (Table 1, Figure 4B)

Both exercise type and humeral elevation angle $(\mathrm{p}<0.01)$ had significant main effects on scapular upward rotation. No significant interaction between exercise type and humeral elevation angle was seen for scapular upward rotation. The scapular upward rotation was significantly greater during the military press than during shoulder flexion with or without weights throughout the entire range of the humeral elevation angles $(\mathrm{p}<$ $0.05)$.

\subsection{Scapular Anterior/Posterior Tilt (Table 1, Figure 4C)}

The type of exercise had a significant main effect $(\mathrm{p}<0.01)$ on the scapular anterior/posterior tilt, with a significant interaction noted between the type of exercise and the humeral elevation angle $(\mathrm{p}<0.01)$. Post-hoc analysis with Holm adjustment revealed that the scapular posterior tilt was significantly greater during the military press than during shoulder flexion at angles from $40^{\circ}$ to $120^{\circ}$ and from $60^{\circ}$ to $100^{\circ}$ for shoulder flexion with and without 2-kg weights, respectively.

\subsection{Clavicular retraction/protraction (Table 2, Figure 5A)}

The type of exercise significantly affected clavicular retraction $(\mathrm{p}<0.01)$, with a significant interaction noted between the type of exercise and the humeral elevation angle $(\mathrm{p}<0.01)$. Clavicular retraction was significantly greater during the military press than 
Scapula and clavicle kinematic characteristics of military press

183

184

185

186

187

188

189

190

191

192

193 during shoulder flexion with weights at humeral elevation angles from $30^{\circ}$ to $100^{\circ}$ and significantly greater than during shoulder flexion without weights at humeral elevation angles of $30^{\circ}$ to $120^{\circ}(\mathrm{p}<0.05)$.

3.5. Clavicular elevation/depression (Table 2, Figure 5B)

The type of exercise significantly affected the clavicular elevation angle $(\mathrm{p}<0.01)$, with a significant interaction noted between the type of exercise and the humeral elevation angle $(\mathrm{p}<0.01)$. Clavicular elevation was significantly greater during the military press than during shoulder flexion at humeral elevation angles of $30^{\circ}$ to $80^{\circ}$ and $120^{\circ}$ for shoulder flexion without weights $(\mathrm{p}<0.05)$ but only at humeral elevation angles of $40^{\circ}$ to $70^{\circ}$ and $120^{\circ}$ for shoulder flexion with weights $(\mathrm{p}<0.05)$. 
Scapula and clavicle kinematic characteristics of military press

194

195

196

197

198

199

200

201

202

203

204

205

206

207

208

209

210

211

212

213

214

215

216

217

218

\section{Discussion}

The three-dimensional kinematic features of the military press were compared with those of shoulder flexion performed with and without weights to clarify the characteristics of this exercise. The military press involved greater scapular upward rotation, posterior tilt, and external rotation relative to normal shoulder flexion in at least part of the examined range, which supported our hypothesis.

Several previous studies have evaluated the kinematics of the scapula and clavicle during humeral elevation. $8,16,18,28$ The results of the present study agree with those of the previous studies.

However, detailed examination revealed that the scapular motions during the military press differed from those during shoulder flexion either with or without weights, and involved less scapular internal rotation, greater posterior tilt, and greater upward rotationwhichwere noted mainly in the initial and middle range of shoulder elevation.

The military press also produced greater clavicular elevation and retraction. The differences between the military press and shoulder flexion became smaller as the angle of elevation increased, and no significant difference except in scapular upward rotation was noted for scapular and clavicular motion at angles $>110^{\circ}$.

Previous studies using EMG have revealed that the trapezius, especially the upper trapezius, and serratus anterior muscles are highly activated during the military press. ${ }^{2122}$ The serratus anterior muscle is known to be responsible for posterior tilt, external rotation, and upward rotation of the scapula, ${ }^{5,13}$ and these actions are consistent with the scapular motions seen during the military press in the present study. The upper trapezius is known to elevate the clavicle ${ }^{27}$, and this action is also consistent with the greater clavicular elevation during the military press revealed in this study.

Clavicular motion is known to relate directly to scapular translation with respect to 
Scapula and clavicle kinematic characteristics of military press

the thorax. Given the lack of significant motion at the acromioclavicular joint, clavicular elevation and retraction will translate the scapula superiorly and posteriorly, i.e., elevation and retraction. ${ }^{12}$ Together with the three motions of the scapula, these two scapular translations account for the five degrees of freedom of scapular motion. ${ }^{20}$

Another kinematic difference between shoulder flexion and the military press was the inclusion of elbow extension and flexion during shoulder motion. The combined shoulder and elbow motion used for the military press is probably more similar to motions used in daily activities, such as reaching up or putting something on a shelf, and may therefore be more "functional."

In this study, the maximum angular differences in scapular position observed between the military press and flexion with $2-\mathrm{kg}$ weights were $7.5^{\circ}$ less internal rotation, $4.1^{\circ}$ greater upward rotation, and $7.6^{\circ}$ greater posterior tilt in the military press. Ludewig et al. ${ }^{14}$ have reported on the kinematic differences between subjects with impingement and control subjects. They observed significant differences in all three rotations of the scapula, with greater medial rotation, greater anterior tilt, and less upward rotation in the impingement group. The maximum mean differences were $5.2^{\circ}$ for medial rotation, $5.8^{\circ}$ for anterior tilt, and $4.1^{\circ}$ for upward rotation. They concluded that a modest angular difference of $4-6^{\circ}$ was sufficient to produce clinically relevant changes in subacromial space and impingement. As the maximum differences observed in our study were above this threshold, we consider the differences in scapular position demonstrated herein to be clinically meaningful.

\section{Use of the military press as a coordination exercise}


Scapula and clavicle kinematic characteristics of military press

The characteristics of scapular and clavicular motion observed during the military press might be useful as a shoulder coordination exercise as part of a rehabilitation program. Many previous studies have reported ${ }^{6,9,14,17}$ decreased scapular external rotation, scapular upward rotation, and posterior scapular tilting in patients with shoulder impingement syndrome, and a similar pattern has also been seen in subjects with glenohumeral instability. ${ }^{11,23,25,30,31}$ A study by Oyama et al. ${ }^{24}$ measured the three-dimensional motion of the scapula and the clavicle while performing various exercises, in the prone position, that retract (externally rotate) the scapula; the results suggested that these exercises could be effective for restoring normal scapular and clavicular kinematics and might be indicated for patients with shoulder pathologies. The military press also involves external rotation accompanied by upward rotation and posterior tilting of the scapula. Moreover, because the military press employs the desired motion of the scapula as part of a more practical motion - i.e., humeral elevation involving the movement of multiple joints - it may be a useful re-education exercise for patients with pathologic conditions characterized by decreased scapular external rotation, decreased scapular upward rotation, and decreased posterior scapular tilting.

Some limitations of the present study should be considered. First, due to the requirements for accurate measurement using the electromagnetic sensor, the data corresponding to shoulder flexion angles of $>120^{\circ}$ were not analyzed. Second, the subjects in this study were healthy young men. Therefore, the results may not be directly applicable to patients with shoulder problems. Third, the military press in this study was performed in the sagittal plane. It cannot be assumed that the results of this study will be applicable to other forms of the military press. Finally, the efficiency of the exercise in improving scapular kinematics has not been determined. Further study is needed in subjects with shoulder pathology and to determine the effectiveness of the exercise. 
Scapula and clavicle kinematic characteristics of military press

267

268

269

270

271

272

273

274

275

276

277

278

279

280

281

282

283

284

285

286

287

288

289

290

\section{Conclusions}

The three-dimensional kinematic characteristics of the scapular and clavicular movements during the military press were investigated and compared with those during shoulder flexion. The military press produced greater upward rotation, external rotation, and posterior tilting of the scapula and more protraction and elevation of the clavicle.

These kinematic features of the military press may make it a useful re-education exercise for patients with decreased scapular external rotation, upward rotation, and posterior tilting. This study may serve as a kinematic basis for prescribing this well-known exercise in clinical practice.

\section{References}

1.Borstad JD, Ludewig PM. Comparison of scapular kinematics between elevation and lowering of the arm in the scapular plane. Clin Biomech (Bristol, Avon) 2002; 17:650-659.

2.Bull M, Ferreira M, Vitti M. Electromyographic validation of the muscles deltoid (anterior portion) and pectoralis major (clavicular portion) in military press exercises with middle grip. J. Morphol. Sci. 2011; 28: 240-245.

3.Bull M, Ferreira M, Vitti M. Electromyographic validation of the deltoid (anterior portion) and pectoralis major (clavicular portion) in military press exercises with open and middle grips. J. Morphol. Sci. 2011; 28:287-289.

4.Crenshaw k, Harmon k, Reed J, Donatucci D. Conditioning of the shoulder complex for specific sports. Philadelphia: Churchill Livingstone Elsevier; 2009. (ISBN No. 0443067015) 


\section{Scapula and clavicle kinematic characteristics of military press}

291

292

293

294

295

296

297

298

299

300

301

302

303

304

305

306

307

308

309

310

5.Ebaugh DD, McClure PW, Karduna AR. Three-dimensional scapulothoracic motion during active and passive arm elevation. Clin Biomech (Bristol, Avon) 2005; 20:700-709. 10.1016/j.clinbiomech.2005.03.008.

6.Endo K, Ikata T, Katoh S, Takeda Y. Radiographic assessment of scapular rotational tilt in chronic shoulder impingement syndrome. J Orthop Sci 2001; 6:3-10.

7.Escamilla RF, Yamashiro K, Paulos L, Andrews JR. Shoulder muscle activity and function in common shoulder rehabilitation exercises. Sports Med 2009; 39:663-685. 10.2165/00007256-200939080-00004.

8.Fung M, Kato S, Barrance PJ, Elias JJ, McFarland EG, Nobuhara K et al. Scapular and clavicular kinematics during humeral elevation: a study with cadavers. J Shoulder Elbow Surg 2001; 10:278-285. 10.1067/mse.2001.114496.

9.Hebert LJ, Moffet H, McFadyen BJ, Dionne CE. Scapular behavior in shoulder impingement syndrome. Arch Phys Med Rehabil 2002; 83:60-69.

10.Horrigan JM, Shellock FG, Mink JH, Deutsch AL. Magnetic resonance imaging evaluation of muscle usage associated with three exercises for rotator cuff rehabilitation. Med Sci Sports Exerc 1999; 31:1361-1366.

11.Illyes A, Kiss RM. Kinematic and muscle activity characteristics of multidirectional shoulder joint instability during elevation. Knee Surg Sports Traumatol Arthrosc 2006; 14:673-685. 10.1007/s00167-005-0012-7.

12.Karduna AR, McClure PW, Michener LA, Sennett B. Dynamic measurements of three-dimensional scapular kinematics: a validation study. J Biomech Eng 2001; 123:184-190.

13.Ludewig PM, Cook TM, Nawoczenski DA. Three-dimensional scapular orientation and 


\section{Scapula and clavicle kinematic characteristics of military press}

muscle activity at selected positions of humeral elevation. J Orthop Sports Phys Ther 1996; 24:57-65.

14.Ludewig PM, Cook TM. Alterations in shoulder kinematics and associated muscle activity in people with symptoms of shoulder impingement. Phys Ther 2000; 80:276-291.

15.Ludewig PM, Behrens SA, Meyer SM, Spoden SM, Wilson LA. Three-dimensional clavicular motion during arm elevation: reliability and descriptive data. J Orthop Sports Phys Ther $2004 ; 34: 140-149$.

16.Ludewig PM, Phadke V, Braman JP, Hassett DR, Cieminski CJ, LaPrade RF. Motion of the shoulder complex during multiplanar humeral elevation. J Bone Joint Surg Am 2009; 91:378-389. 10.2106/JBJS.G.01483.

17.Lukasiewicz AC, McClure P, Michener L, Pratt N, Sennett B. Comparison of 3-dimensional scapular position and orientation between subjects with and without shoulder impingement. J Orthop Sports Phys Ther 1999; 29:574-583+ADs- discussion 584-576.

18.McClure PW, Michener LA, Sennett BJ, Karduna AR. Direct 3-dimensional measurement of scapular kinematics during dynamic movements in vivo. J Shoulder Elbow Surg 2001; 10:269-277. 10.1067/mse.2001.112954.

19.McQuade KJ, Smidt GL. Dynamic scapulohumeral rhythm: the effects of external resistance during elevation of the arm in the scapular plane. J Orthop Sports Phys Ther 1998; 27:125-133.

20.Michener LA, McClure PW, Karduna AR. Anatomical and biomechanical mechanisms of subacromial impingement syndrome. Clin Biomech (Bristol, Avon) 2003; 18:369-379.

21.Moseley JB, Jr., Jobe FW, Pink M, Perry J, Tibone J. EMG analysis of the scapular muscles during a shoulder rehabilitation program. Am J Sports Med 1992; 20:128-134. 


\section{Scapula and clavicle kinematic characteristics of military press}

337

338

339

340

341

342

343

22.Nagai K, Tateuchi H, Takashima S, Miyasaka J, Hasegawa S, Arai R et al. Effects of trunk rotation on scapular kinematics and muscle activity during humeral elevation. J Electromyogr Kinesiol 2013. 10.1016/j.jelekin.2013.01.012.

23. Ogston JB, Ludewig PM. Differences in 3-dimensional shoulder kinematics between persons with multidirectional instability and asymptomatic controls. Am J Sports Med 2007; 35:1361-1370. 10.1177/0363546507300820.

24.Oyama S, Myers JB, Wassinger CA, Lephart SM. Three-dimensional scapular and clavicular kinematics and scapular muscle activity during retraction exercises. J Orthop Sports Phys Ther 2010; 40:169-179. 10.2519/jospt.2010.3018.

25.Ozaki J. Glenohumeral movements of the involuntary inferior and multidirectional instability. Clin Orthop Relat Res 1989:107-111.

26.Paoli A, Marcolin G, Petrone N. Influence of different ranges of motion on selective recruitment of shoulder muscles in the sitting military press: an electromyographic study. $\mathrm{J}$ Strength Cond Res 2010; 24:1578-1583. 10.1519/JSC.0b013e3181d756ea.

27.Pratt NE. Anatomy and Biomechanics of the Shoulder. Journal of Hand Therapy 1994; 7:65-76. 10.1016/s0894-1130(12)80074-3.

28.Teece RM, Lunden JB, Lloyd AS, Kaiser AP, Cieminski CJ, Ludewig PM. Three-dimensional acromioclavicular joint motions during elevation of the arm. J Orthop Sports Phys Ther 2008; 38:181-190. 10.2519/jospt.2008.2386.

29.Townsend H, Jobe FW, Pink M, Perry J. Electromyographic analysis of the glenohumeral muscles during a baseball rehabilitation program. Am J Sports Med 1991; 19:264-272.

30.von Eisenhart-Rothe R, Matsen FA, 3rd, Eckstein F, Vogl T, Graichen H. Pathomechanics in atraumatic shoulder instability: scapular positioning correlates with humeral head 
Scapula and clavicle kinematic characteristics of military press

360

361

centering. Clin Orthop Relat Res 2005:82-89.

31.Warner JJ, Micheli LJ, Arslanian LE, Kennedy J, Kennedy R. Scapulothoracic motion in normal shoulders and shoulders with glenohumeral instability and impingement syndrome. A study using Moire topographic analysis. Clin Orthop Relat Res 1992:191-199.

32.Wu G, van der Helm FC, Veeger HE, Makhsous M, Van Roy P, Anglin C et al. ISB recommendation on definitions of joint coordinate systems of various joints for the reporting of human joint motion--Part II: shoulder, elbow, wrist and hand. J Biomech 2005; 38:981-992.

\section{Figure Legend}

Figure 1

The locations of the anatomic landmarks used for digitization and to establish the coordinate axes

Thorax: C7, the spinous process of the seventh cervical vertebra; $\mathrm{T} 8$, the spinous process of the eighth cervical vertebra; SN, sternal notch; XP, xiphoid process. Scapula: AC, acromioclavicular joint; BS, base of spine; IA, inferior angle. Humerus: ME, medial epicondyle; LE, lateral epicondyle. Clavicle: ACl, acromioclavicular joint; SC, sternoclavicular joint.

\section{Figure 2}

Shoulder flexion with and without weights and the military press

Figure 3

Definitions of the motions of the scapula and clavicle

A: Upward-downward scapular rotation as seen in the posterior view of a right shoulder; B: 
Scapula and clavicle kinematic characteristics of military press

385

386

387

388

389

390

391

392

393

394

395

396

397

398

399

400

401

402

403

404

405

internal-external scapular rotation as seen in the superior view of a right shoulder; C: anterior-posterior scapular tilting as seen in the lateral view of a right shoulder; D: clavicular retraction-protraction as seen in the superior view of a right shoulder; E: clavicular elevation-depression as seen in the anterior view of a right shoulder.

Figure 4

The motions of the scapula relative to the thorax during humeral elevation.

A: scapular internal/external rotation; B: scapular upward/downward rotation; C: scapular posterior/anterior tilting. Circles: military press; squares, flexion with 2-kg weights; triangles, flexion without weights

Figure 5

The motions of the clavicle relative to the thorax during humeral elevation.

A: clavicular retraction/protraction; B: clavicular elevation/depression. Circles: military press; squares: flexion with 2-kg weights; triangles: flexion without weights

\section{Table 1}

Changes in the scapular position at each humeral elevation angle.

The values are expressed as the mean angle \pm standard deviation.

\section{Table 2}

Changes in the clavicular position at each humeral elevation angle.

The values are expressed as the mean angle \pm standard deviation. 

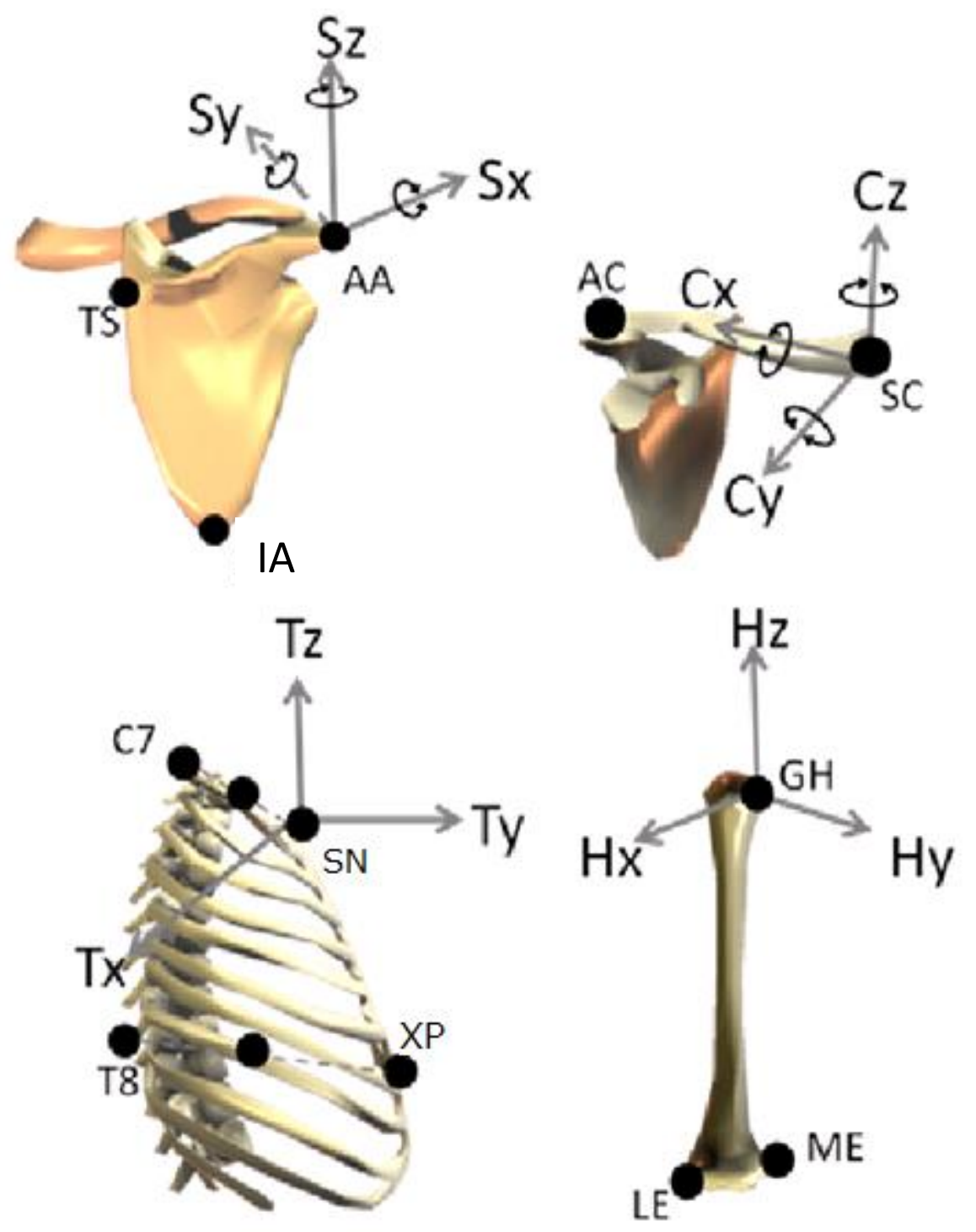

Figure 1 


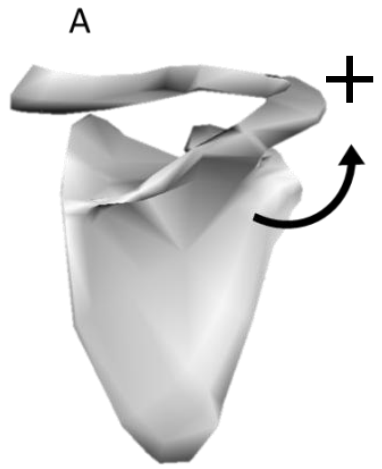

Upward Rotation

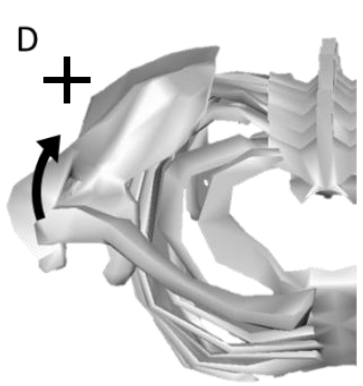

Retraction
B

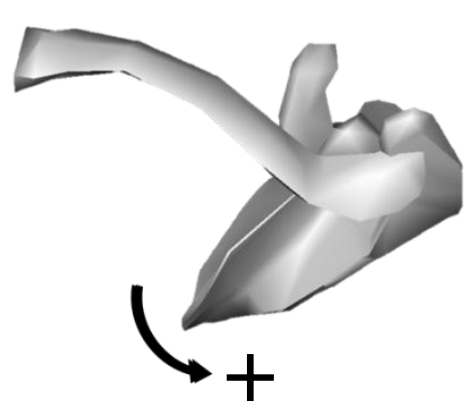

Internal Rotation

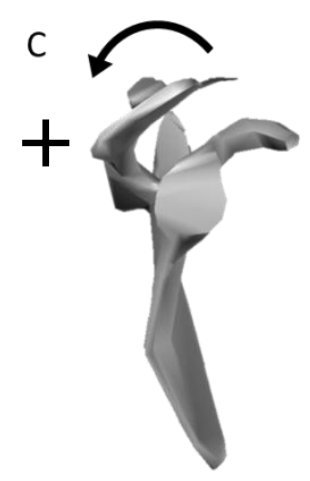

Posterior Tilting

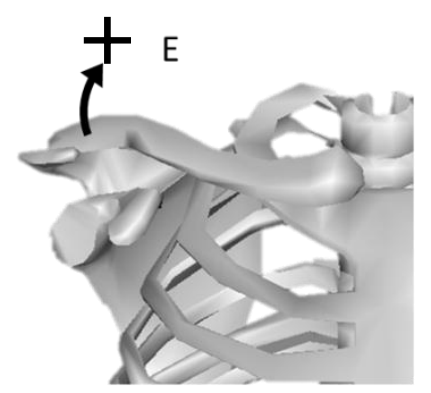

Elevation

Figure 2 


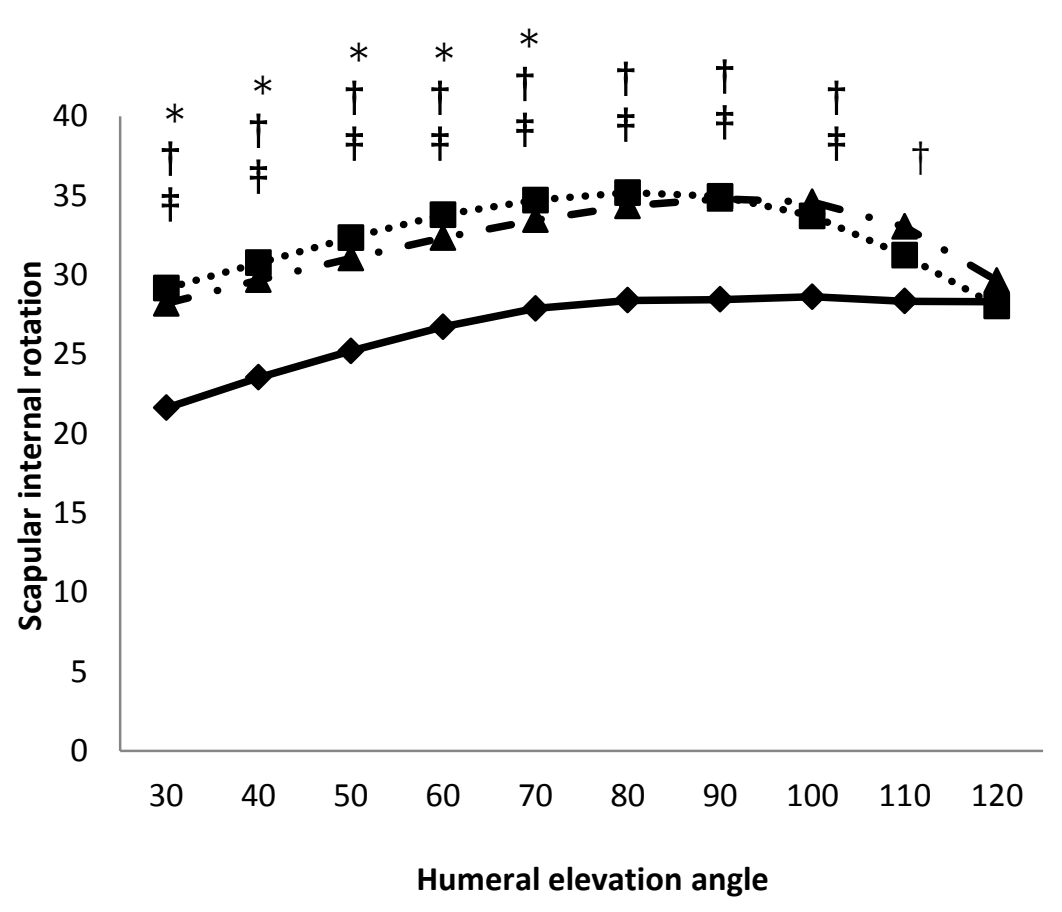

*: significant difference between flexion without weights and with 2-kg weights ( $p<$ 0.05) 0.05)

$\dagger$ : significant difference between flexion without weights and the military press $(p<$

‡: significant difference between flexion with 2-kg weights and the military press $(p<$ 0.05)

Figure $3 \mathrm{~A}$ 


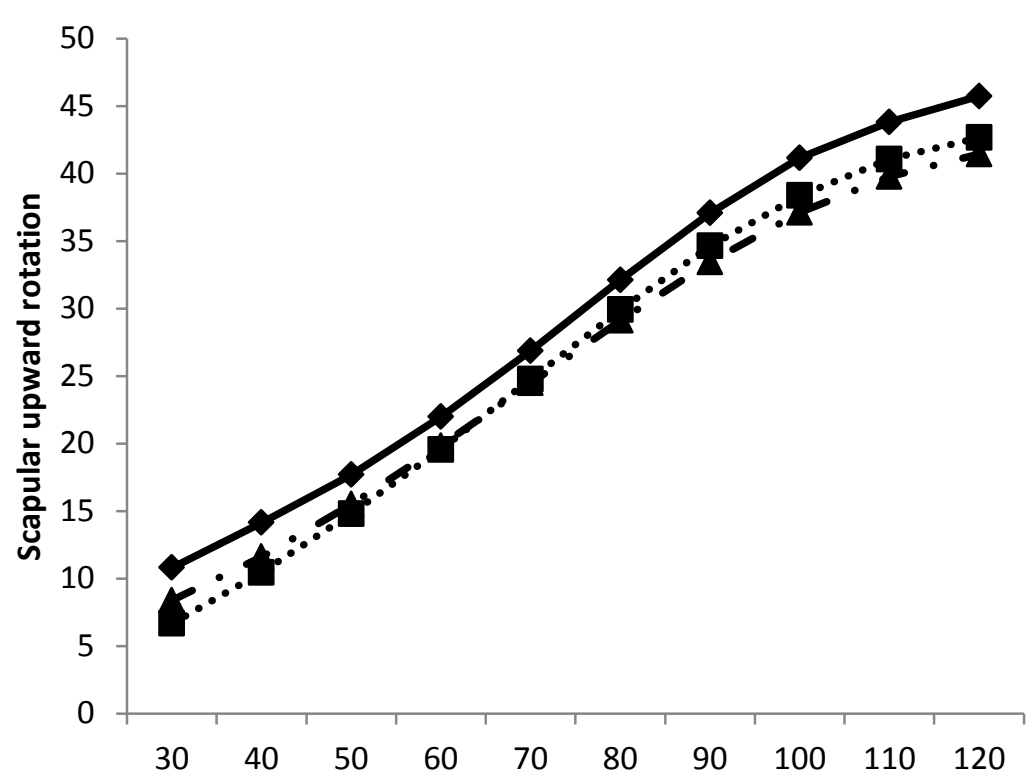

Post-hoc analysis of the main effect (exercise type) flexion without weight-flexion with 2-kg weights: $p=1.00$ flexion without weight-military press: $p<0.05$ flexion with 2-kg weights-military press: $p<0.05$

Humeral elevation angle

Figure $3 \mathrm{~B}$ 


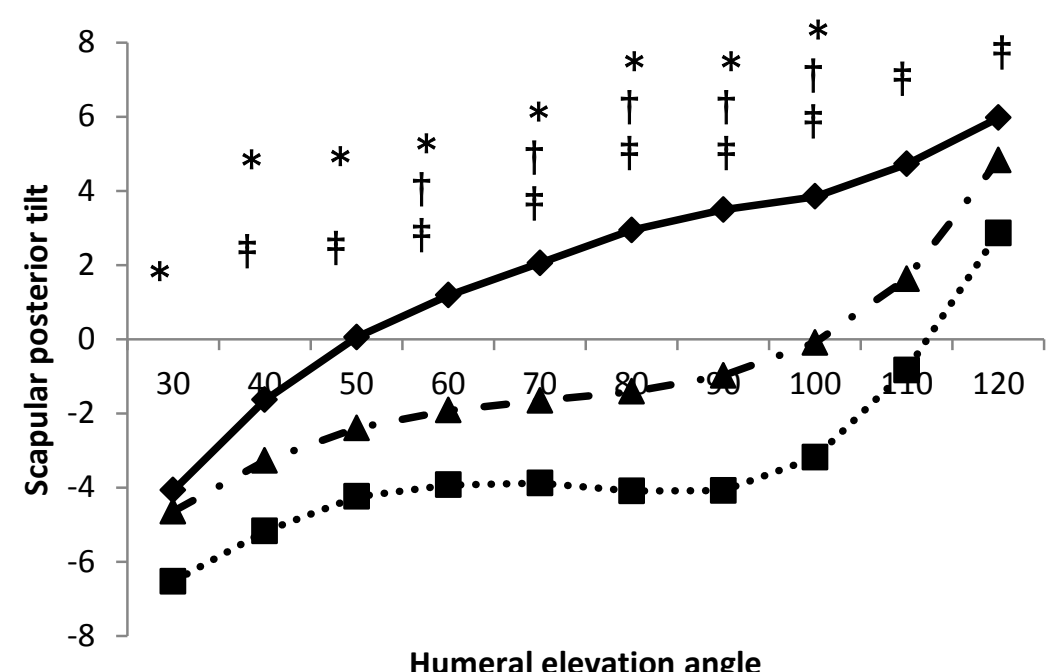

-4. Flexion without weights

..-. Flexion with 2-kg weights

$\multimap$ Military press

*: significant difference between flexion without weights and with $2-\mathrm{kg}$ weights ( $p<$ 0.05)

$\dagger$ : significant difference between flexion without weights and the military press $(p<$ 0.05)

‡: significant difference between flexion with 2-kg weights and the military press ( $p<$ 0.05)

Figure $3 \mathrm{C}$ 


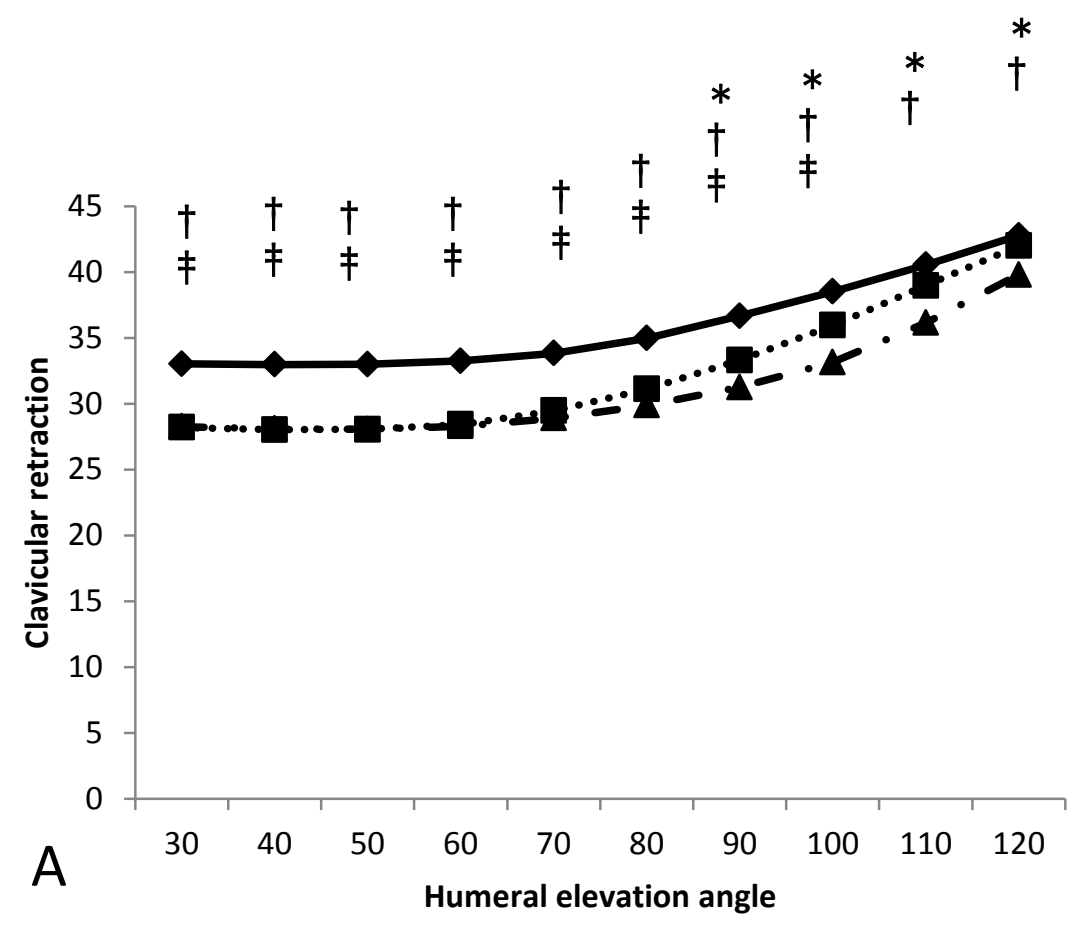
0.05)

*: significant difference between flexion without weights and with 2-kg weights $(p<$

$\dagger$ : significant difference between flexion without weights and the military press $(p<$ 0.05)

キ: significant difference between flexion with 2-kg weights and the military press ( $p<$ 0.05)

Figure $4 \mathrm{~A}$ 


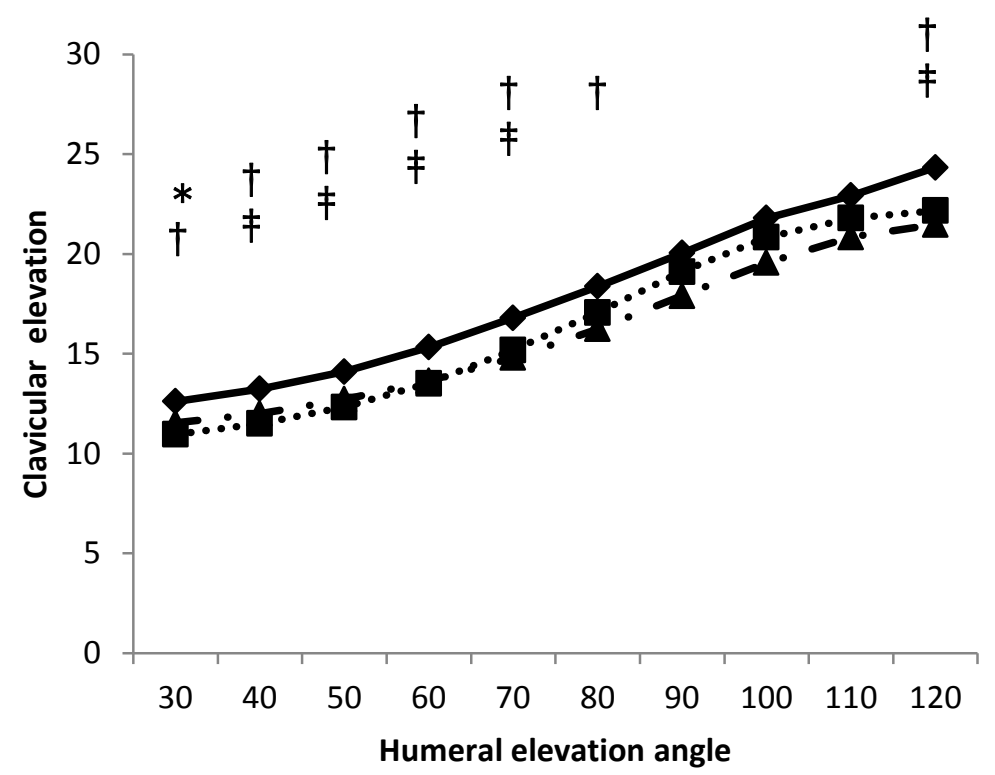
$0.05)$

*: significant difference between flexion without weights and with 2-kg weights ( $p<$ 0.05)

₹: significant difference between flexion with 2-kg weights and the military press ( $p<$ 0.05)
-^. Flexion without weights

.... Flexion with 2-kg weights

$\rightarrow$ Military press

Figure 4 B 


\begin{tabular}{|c|c|c|c|c|c|c|c|c|c|c|c|}
\hline & & $30^{\circ}$ & $40^{\circ}$ & $50^{\circ}$ & $60^{\circ}$ & $70^{\circ}$ & $80^{\circ}$ & $90^{\circ}$ & $100^{\circ}$ & $110^{\circ}$ & $120^{\circ}$ \\
\hline \multirow{4}{*}{$\begin{array}{l}\text { internal } \\
\text { rotation }\end{array}$} & Flexion without weight & $28.2 \pm 10.2$ & $29.7 \pm 10.4$ & $31.1 \pm 10.8$ & $32.4 \pm 11.2$ & $33.5 \pm 11.6$ & $34.3 \pm 12.1$ & $34.8 \pm 12.5$ & $34.6 \pm 13.0$ & $33.0 \pm 13.3$ & $29.6 \pm 14.2$ \\
\hline & Flexion with $2 \mathrm{~kg}$ weight & $29.2 \pm 10.1$ & $30.8 \pm 10.3$ & $32.4 \pm 10.6$ & $33.8 \pm 11.0$ & $34.7 \pm 11.4$ & $35.2 \pm 11.9$ & $34.9 \pm 12.6$ & $33.7 \pm 13.2$ & $31.2 \pm 14.0$ & $28.0 \pm 15.1$ \\
\hline & Military press & $21.6 \pm 11.1$ & $23.5 \pm 11.1$ & $25.2 \pm 11.4$ & $26.7 \pm 12.0$ & $27.9 \pm 12.5$ & $28.4 \pm 12.9$ & $28.5 \pm 12.9$ & $28.6 \pm 12.8$ & $28.3 \pm 12.6$ & $28.3 \pm 13.6$ \\
\hline & & $30^{\circ}$ & $40^{\circ}$ & $50^{\circ}$ & $60^{\circ}$ & $70^{\circ}$ & $80^{\circ}$ & $90^{\circ}$ & $100^{\circ}$ & $110^{\circ}$ & $120^{\circ}$ \\
\hline \multirow{4}{*}{$\begin{array}{l}\text { upward } \\
\text { rotation }\end{array}$} & Flexion without weight & $8.4 \pm 7.0$ & $11.6 \pm 7.0$ & $15.5 \pm 7.2$ & $19.8 \pm 7.4$ & $24.5 \pm 7.6$ & $29.1 \pm 7.7$ & $33.4 \pm 7.7$ & $37.1 \pm 7.5$ & $39.8 \pm 7.2$ & $41.4 \pm 7.1$ \\
\hline & Flexion with $2 \mathrm{~kg}$ weight & $6.7 \pm 7.0$ & $10.5 \pm 6.9$ & $14.8 \pm 7.2$ & $19.5 \pm 7.5$ & $24.8 \pm 7.8$ & $29.9 \pm 8.1$ & $34.6 \pm 8.2$ & $38.4 \pm 8.1$ & $41.1 \pm 7.9$ & $42.7 \pm 7.5$ \\
\hline & Military press & $10.8 \pm 8.2$ & $14.1 \pm 8.0$ & $17.7 \pm 8.3$ & $22.0 \pm 8.7$ & $26.9 \pm 8.9$ & $32.1 \pm 9.1$ & $37.1 \pm 9.2$ & $41.2 \pm 8.8$ & $43.8 \pm 8.3$ & $45.7 \pm 7.7$ \\
\hline & & $30^{\circ}$ & $40^{\circ}$ & $50^{\circ}$ & $60^{\circ}$ & $70^{\circ}$ & $80^{\circ}$ & $90^{\circ}$ & $100^{\circ}$ & $110^{\circ}$ & $120^{\circ}$ \\
\hline \multirow{3}{*}{$\begin{array}{c}\text { posterior } \\
\text { tilt }\end{array}$} & Flexion without weight & $-4.6 \pm 6.0$ & $-3.3 \pm 6.5$ & $-2.4 \pm 6.9$ & $-1.9 \pm 7.5$ & $-1.7 \pm 8.3$ & $-1.4 \pm 9.3$ & $-1.0 \pm 10.3$ & $-0.1 \pm 11.2$ & $1.6 \pm 12.2$ & $4.8 \pm 13.6$ \\
\hline & Flexion with $2 \mathrm{~kg}$ weight & $-6.5 \pm 6.3$ & $-5.2 \pm 6.4$ & $-4.2 \pm 6.7$ & $-3.9 \pm 7.3$ & $-3.9 \pm 8.2$ & $-4.1 \pm 9.3$ & $-4.1 \pm 10.1$ & $-3.2 \pm 11.1$ & $-0.8 \pm 12.5$ & $2.9 \pm 14.0$ \\
\hline & Military press & $-4.1 \pm 7.7$ & $-1.6 \pm 7.6$ & $0.1 \pm 7.9$ & $1.2 \pm 8.3$ & $2.1 \pm 9.0$ & $2.9 \pm 9.6$ & $3.5 \pm 10.5$ & $3.8 \pm 12.0$ & $4.7 \pm 13.2$ & $6.0 \pm 14.5$ \\
\hline
\end{tabular}

\section{Table 1 . Scapular position changes in each humeral elevation angle}




\begin{tabular}{|c|c|c|c|c|c|c|c|c|c|c|c|}
\hline & & $30^{\circ}$ & $40^{\circ}$ & $50^{\circ}$ & $60^{\circ}$ & $70^{\circ}$ & $80^{\circ}$ & $90^{\circ}$ & $100^{\circ}$ & $110^{\circ}$ & $120^{\circ}$ \\
\hline \multirow{4}{*}{$\begin{array}{l}\text { clavicular } \\
\text { retraction }\end{array}$} & Flexion without weight & $28.3 \pm 7.4$ & $28.1 \pm 7.6$ & $28.1 \pm 7.9$ & $28.3 \pm 8.1$ & $28.9 \pm 8.3$ & $29.9 \pm 8.4$ & $31.3 \pm 8.4$ & $33.2 \pm 8.2$ & $36.2 \pm 7.8$ & $39.8 \pm 7.6$ \\
\hline & Flexion with $2 \mathrm{~kg}$ weight & $28.2 \pm 7.5$ & $28.0 \pm 7.8$ & $28.1 \pm 8.1$ & $28.4 \pm 8.3$ & $29.5 \pm 8.3$ & $31.1 \pm 8.3$ & $33.3 \pm 8.3$ & $36.0 \pm 8.2$ & $39.0 \pm 8.1$ & $42.0 \pm 8.0$ \\
\hline & Military press & $33.0 \pm 8.2$ & $33.0 \pm 8.5$ & $33.0 \pm 8.8$ & $33.3 \pm 9.0$ & $33.8 \pm 9.1$ & $35.0 \pm 9.0$ & $36.7 \pm 8.8$ & $38.5 \pm 8.6$ & $40.5 \pm 8.3$ & $42.8 \pm 8.2$ \\
\hline & & $30^{\circ}$ & $40^{\circ}$ & $50^{\circ}$ & $60^{\circ}$ & $70^{\circ}$ & $80^{\circ}$ & $90^{\circ}$ & $100^{\circ}$ & $110^{\circ}$ & $120^{\circ}$ \\
\hline \multirow{3}{*}{$\begin{array}{l}\text { clavicular } \\
\text { elevation }\end{array}$} & Flexion without weight & $11.5 \pm 5.1$ & $12.0 \pm 5.1$ & $12.7 \pm 5.2$ & $13.6 \pm 5.3$ & $14.8 \pm 5.4$ & $16.2 \pm 5.5$ & $17.9 \pm 5.6$ & $19.6 \pm 5.7$ & $20.8 \pm 5.8$ & $21.5 \pm 6.1$ \\
\hline & Flexion with $2 \mathrm{~kg}$ weight & $11.0 \pm 5.1$ & $11.5 \pm 5.2$ & $12.3 \pm 5.4$ & $13.5 \pm 5.8$ & $15.2 \pm 6.1$ & $17.1 \pm 6.4$ & $19.1 \pm 6.7$ & $20.8 \pm 7.0$ & $21.8 \pm 7.2$ & $22.2 \pm 7.5$ \\
\hline & Military press & $12.6 \pm 6.1$ & $13.2 \pm 6.2$ & $14.1 \pm 6.3$ & $15.3 \pm 6.6$ & $16.8 \pm 6.9$ & $18.4 \pm 7.1$ & $20.0 \pm 7.5$ & $21.8 \pm 7.7$ & $22.9 \pm 7.8$ & $24.3 \pm 7.8$ \\
\hline
\end{tabular}

\section{Table 2 . Clavicular position changes in each humeral elevation angle}

